Article

\title{
In Vitro Efficient Expansion of Tumor Cells Deriving from Different Types of Human Tumor Samples
}

Ilaria Turin ${ }^{1}$, Roberta Schiavo ${ }^{2}$, Marcello Maestri ${ }^{3}$, Ombretta Luinetti ${ }^{4}$, Barbara Dal Bello ${ }^{4}$, Marco Paulli ${ }^{4,5}$, Paolo Dionigi ${ }^{3,5}$, Marianna Roccio ${ }^{6}$, Arsenio Spinillo ${ }^{5,6}$, Federica Ferulli ${ }^{1}$, Matteo Tanzi ${ }^{1}$, Rita Maccario ${ }^{7}$, Daniela Montagna ${ }^{1,5, \dagger, *}$ and Paolo Pedrazzoli ${ }^{8, \uparrow}$

1 Laboratorio di Immunologia dei Trapianti, Oncoematologia Pediatrica, Fondazione IRCCS Policlinico S. Matteo, Pavia 27100, Italy; E-Mails: i.turin@smatteo.pv.it (I.T.); federica.ferulli@gmail.com (F.F.); matteo.tanzi01@universitadipavia.it (M.T.)

2 U.O. Microbiologia, Ospedale Guglielmo Da Saliceto, Piacenza 29100, Italy; E-Mail: roberta.schiavo@gmail.com

3 Chirurgia Generale I Fondazione IRCCS Policlinico S. Matteo, Pavia 27100, Italy; E-Mails: m.maestri@smatteo.pv.it (M.M.); paolo.dionigi@unipv.it (P.D.)

4 U.C. Anatomia Patologica, Fondazione IRCCS Policlinico S. Matteo, Pavia 27100, Italy; E-Mails: o.luinetti@smatteo.pv.it (O.L.); b.dalbello@smatteo.pv.it (B.D.B.); marco.paulli@unipv.it (M.P.)

5 Dipartimento di Scienze Clinico-chirurgiche, Pediatriche e Diagnostiche, Università di Pavia, Pavia 20100, Italy; E-Mail: a.spinillo@smatteo.pv.it

6 S.C. Ostetricia e Ginecologia, Fondazione IRCCS Policlinico S. Matteo, Pavia 27100, Italy; E-Mail: m.roccio@smatteo.pv.it

7 Cell Factory, Lab. Ricerca, Fondazione IRCCS Policlinico S. Matteo, Pavia 27100, Italy; E-Mail: r.maccario@smatteo.pv.it

8 S.C. Oncologia, Fondazione IRCCS Policlinico S. Matteo, Pavia 27100, Italy; E-Mail: p.pedrazzoli@smatteo.pv.it

$\dagger$ Equally contributed as senior authors.

* Author to whom correspondence should be addressed; E-Mail: d.montagna@smatteo.pv.it or daniela.montagna@unipv.it; Tel.: +39-0382-502603; Fax: +39-0382-527976.

Received: 14 November 2013; in revised form: 3 March 2014 / Accepted: 4 March 2014 / Published: 26 March 2014 


\begin{abstract}
Obtaining human tumor cell lines from fresh tumors is essential to advance our understanding of antitumor immune surveillance mechanisms and to develop new ex vivo strategies to generate an efficient anti-tumor response. The present study delineates a simple and rapid method for efficiently establishing primary cultures starting from tumor samples of different types, while maintaining the immuno-histochemical characteristics of the original tumor. We compared two different strategies to disaggregate tumor specimens. After short or long term in vitro expansion, cells analyzed for the presence of malignant cells demonstrated their neoplastic origin. Considering that tumor cells may be isolated in a closed system with high efficiency, we propose this methodology for the ex vivo expansion of tumor cells to be used to evaluate suitable new drugs or to generate tumor-specific cytotoxic $\mathrm{T}$ lymphocytes or vaccines.
\end{abstract}

Keywords: tumor cell line; in vitro model; cell culture; immunomagnetic selection

\title{
1. Introduction
}

Tumor cell lines represent a unique tool for investigating both tumor biology/profiles and the mechanisms underlying tumor responsiveness to anticancer therapies [1-3]. The establishment of tumor cell lines is extremely difficult and the success rate is low and unpredictable [4]. Although tumors exhibit apparent autonomy from normal regulatory control in vivo, they often fail to grow when cultured in vitro [2].

Several different cell types, other than neoplastic cells, may be obtained from tumor samples, including connective-tissue fibroblasts, infiltrating lymphocytes and elements of normal tissue from which the neoplasia arose. When trying to establish a tumor cell line, the major problem is contamination by fibroblasts or other types of endothelial cells, which grow readily in culture and may also respond to tumor-derived mitogenic factors [5]. Furthermore, in primary tumor cultures many cells may not be capable of propagation due to genetic or phenotypic aberrations, terminal differentiation or nutritional insufficiency. Nevertheless, some primary cultures can be subcultured, opening up major research possibilities [6,7].

Even though established cell lines are commercially available from a number of sources, there is a need for simple procedures to generate solid tumor cell lines that retain the characteristics of the original tumor. This would help to define, for example tumor associated antigens (TAAs) as an aid in developing immunotherapeutic approaches [8-10].

We report on the development of an efficient methodology to establish primary cultures from tumor tissues and demonstrate how a few simple modifications in cell culture establishment allow the generation of several cell lines from different types of human tumors. 


\section{Materials and Methods}

\subsection{Tumor Samples}

Samples were obtained from surgical procedures and neoplastic effusions. In both cases fresh tumor material was collected in accordance with a protocol approved by the local Ethics Committee. The vast majority of tumor samples were derived from metastasis (99 out of 115 samples). Surgical materials not required for histopathologic diagnosis, were placed in sterile tubes containing RPMI 1640 supplemented with 10\% heat-inactivated fetal bovine serum (FBS), $200 \mathrm{U} / \mathrm{mL}$ penicillin and $200 \mu \mathrm{g} / \mathrm{mL}$ streptomycin (all from Life Technologies Inc., Paisley, UK). In case of neoplastic effusion, tumor cells were recovered by centrifugation. When possible, a portion of the tumor sample was cryopreserved in freezing medium containing 90\% FBS and 10\% dimethyl sulphoxide (DMSO) (Wak-Chemie Medical GMBH, Steinbach/TS, Germany), and stored in liquid nitrogen for further studies.

\subsection{Establishment of Primary Cell Cultures from Surgical Samples}

Two different approaches to obtain viable tumor cells from tumor specimens were utilized, the first included mechanical disaggregation with a cell scraper, while the second, representing an improvement of this methodology, included the use of the Gentle MACS Dissociator (Miltenyi Biotec, Bergisch Gladbach, Germany). In the first case, after removal of debris (i.e., fat and necrotic material), surgical samples were cut into 2-4 mm square sections and washed with sterile phosphate buffered saline (PBS, Life Technologies Inc.). Tumor cells were released from the samples by enzymatic treatment with $0.1 \%$ collagenase (Sigma Aldrich, Irvine, UK) and $50 \mathrm{U} / \mathrm{mL}$ dispase (GIBCO/Invitrogen Life Technologies, Carlsbad, CA, USA), in a $37{ }^{\circ} \mathrm{C}$ water bath for at least two hours, agitating every10-20 min. At the end of incubation, the same amount of ice-cold RPMI supplemented with 20\% FBS (GIBCO/Invitrogen Life Technologies) was added, the tumor cell suspension was passed through a $85-\mu$ m nylon mesh cell strainer (Becton Dickinson, San Jose, CA, USA) and the undigested tissues squeezed with a cell scraper (mechanical disaggregation). In the case of soft tumor samples (i.e., liver metastases) we used only mechanical disaggregation until the tumor tissue was completely dispersed.

Tumor specimens processed with the Gentle MACS Dissociator were treated when necessary, with $0.1 \%$ collagenase for an half hour, and then disaggregated using different protocols depending on the tissue characteristics.

After both procedures, tumor cells were passed in filters (Miltenyi Biotec) to remove clusters and then collected by centrifugation at $1000 \mathrm{rpm}$ for $10 \mathrm{~min}$, checked for viability with trypan blue dye exclusion, resuspended at a concentration of $0.5-1 \times 10^{6}$ cells $/ \mathrm{mL}$ of CellGro SCGM (Cell Genix, Freiburg, Germany),supplemented with 20\% FBS, $2 \mathrm{mM} \mathrm{L-glutamine,} 100 \mathrm{U} / \mathrm{mL}$ penicillin and $100 \mu \mathrm{g} / \mathrm{mL}$ streptomycin (complete medium) (all from Life Technologies Inc.) and cultured in $25 \mathrm{~cm}^{2}$ tissue flasks (Corning, Stone Staffordshire, UK) at $37{ }^{\circ} \mathrm{C}$ and $5 \% \mathrm{CO}_{2}$. Viable tumor cells attached to the flask within 12-24 h. At the first medium change, rather than discarding medium containing unattached cells that may grow and provide a backup culture, we put this into a fresh flask. Cultures at $75 \%$ to $100 \%$ confluence were selected for subculture by trypsinization with $0.25 \%$ trypsin 
and $0.02 \%$ EDTA (Life Technologies Inc.) in a calcium/magnesium-free balanced solution. The culture medium was changed twice a week and cellular homogeneity evaluated microscopically every 24-48 h. When possible, early passage and late passage primary cultures were frozen in $90 \%$ FBS and 10\% DMSO and stored in liquid nitrogen for further experiments.

\subsection{Establishment of Primary Cell Cultures from Pleural or Peritoneal Effusions}

Under sterile conditions, neoplastic effusions were transferred to $50 \mathrm{~mL}$ sterile tubes (Corning, Shiphol Rijk, The Netherlands) and centrifuged at $1200 \mathrm{rpm}$ for $10 \mathrm{~min}$. The supernatant was discarded and cells were washed and resuspended in complete medium. After evaluation of viability, the cells were seeded at a density of $3 \times 10^{6}$ cells $/ \mathrm{mL}$ in $25 \mathrm{~cm}^{2}$ tissue culture flasks (Corning) at $37{ }^{\circ} \mathrm{C}$ and $5 \% \mathrm{CO}_{2}$. Culture medium was changed twice a week and cellular homogeneity was evaluated microscopically every $24-48 \mathrm{~h}$. When cultures reached sub-confluence, cells were collected as previously described and subcultured at a dilution of 1:3 until they began to grow rapidly, and then serially subcultured at a dilution of 1:5 every three days.

\subsection{Ascite-Derived Ovarian Cancer Cells}

Approximately $200 \mathrm{~mL}$ of ascitic fluid was obtained from ten patients. Ascitic fluid was transferred to $50 \mathrm{~mL}$ tubes, centrifuged and resuspended in an appropriate volume with PBS (Gibco, Grand Island, NY, USA). After pathology evaluation for the presence of tumor cells, a fraction of the cells was cryopreserved for later use and seeded in $25 \mathrm{~cm}^{2}$ flasks at a concentration of $0.5 \times 10^{6} / \mathrm{mL}$.

Alternatively, mononuclear cells were isolated by Ficoll gradient separation and then incubated with the CD326 (EpCAM) Tumor Cell Enrichment and Detection Kit (Miltenyi Biotec) according to the manufacturer's instructions. Cells isolated from ascitic fluid were phenotypically and histochemically characterized to confirm their neoplastic origin and then cryopreserved or alternatively cultured as described above, and further expanded. In selected cases, when a large amount of cells was available, cell culture purity was improved by negative selection with anti-fibroblast microbeads (MiniMacs, Miltenyi Biotec), performed according to the manufacturer's instructions. At every fifth passage a portion of the cells was frozen in $90 \%$ FBS and 10\% DMSO and stored in liquid nitrogen for further experiments.

\subsection{Phenotypical Analysis}

Two different approaches were employed to evaluate the neoplastic component of cultured cells derived from tumor samples. For cells obtained from the first 75 samples, the morphological and immunocytochemical characteristics of tumor cell lines were studied at the 4-5th passage, and every 20 passages thereafter, by immuno-enzymatic staining with tumor-specific monoclonal antibodies. Cultures were fixed in 10\% neutral-buffered formalin, embedded in paraffin and then stained with haematoxylin-eosin and with periodic acid-Schiff stain (PAS) to define the presence of malignant cells on the basis of cytomorphology. For the identification of specific tumor cells, we tested monoclonal antibodies against cytokeratin, vimentin/Clone V9, CD99 and HER2/neu (Dako, Glostruo, Denmark), EGFR (Neomarkers, Fremont, CA, USA), CD326 (Miltenyi Biotec) using indirect immuno-enzymatic 
staining according to the manufacturers' instructions. Cultured tumor cells from ovarian cancer were also evaluated by cytofluorimetric analysis for the expression of CD326 antigen. Evaluation of CD326 expression was performed by direct immunofluorescence, using phycoerythrin (PE)-anti CD326 specific monoclonal antibody (Miltenyi Biotec), according to previously reported methods (8). Examination of growing cultures was performed using a direct phase-contrast microscope. Alternatively, at least three cytospins were performed using $10^{5}$ cultured cells/cytospin obtained after 3-5 passages, for morphological and immunocytochemical analysis. Cells were fixed in 95\% alcohol and one slide was stained with haematoxylin-eosin to identify malignant cells on the basis of cytomorphology. To distinguish tumor cells from hyperplastic mesothelial cells, the other slides were tested with monoclonal antibodies against cytokeratin CAM 5.2 (Dako) and calretinin (Invitrogen), using indirect immuno-enzymatic staining according to the manufacturers' instructions. Tumor cells were assessed by a semiquantative method.

\section{Results}

\subsection{Generation of Primary Cultures}

Two different methodologies to obtain viable tumor cells from tumor specimens were utilized. The first 75 consecutive tumor samples were treated with mechanical disaggregation after 2-h of enzymatic treatment, while the next 40 were disaggregated with GentleMACS dissociator. Primary culture was considered successful if it met the following criteria: (i) presence of a pure line of tumor cells as confirmed by morphological and immunohistochemical analysis; (ii) sufficient number of cells to perform immunological and molecular studies; (iii) cell viability when they were thawed. By these criteria, we were able to generate 40 primary cell cultures from 75 tumor biopsies (54\%) and 5 primary cultures from 5 neoplastic effusions (100\%). Table 1 summarizes the findings on the 80 specimens included in this part of the study. All primary cultures were maintained for at least three weeks in culture before phenotypical analysis and a portion of early passage cultures was frozen for further studies. In particular, we were able to generate and characterize ten tumor cell lines out of 40 primary cultures. Cultures from metastases were generally more difficult to maintain in culture and we only reached a maximum of 11 passages, while cultures from primary tumors generally grew well and were maintained for 24-30 passages. We also evaluated the average number of passages that various primary cultures could reach in vitro (Figure 1) and observed some differences between tumor types: while ovarian cancer, kidney tumor, breast carcinoma and sarcomas grew quickly and easily reached 20 passages in culture; colorectal cancer, gastrointestinal stromal tumors (GIST) and melanoma were generally more difficult to maintain long term. Cultures starting from a malignant effusion were easily established and maintained an average of at least 14 passages (Figure 1). In seven cultures (2 sarcoma, 3 kidney and 2 ovary) after 3 months of culture, we observed a shorter doubling time (compared with the initial one), possibly related to a spontaneous immortalization process.

The introduction of GentleMACS for tumor fragment disaggregation greatly improved the capacity to establish tumor cell lines, irrespective of their origin (primary tumor or metastasis). From 40 tumor fragments (4 derived from a primary tumor and 36 from metastases) we were able to establish 31 tumor cell lines ( 3 from primary tumor and 28 from metastases) with an efficiency of about $77 \%$ 
(Table 2). Cell recovery after each passage was in general, much higher than that obtained when we performed disaggregation with a cell scraper. In this case, considering the high recovery of tumor cells, we decided to limit the expansion of cell cultures to the first 3-5 passages, to avoid any possible mutation of tumor cells after long-term culture. Thus after each passage, a portion of the tumor cells were cryopreserved, while the remaining cells were seeded. In all cases, evaluation of phenotypic features of the cells did not reveal differences between the different passages (data not shown). Statistical analysis using the Fisher's Exact Text to compare the proportion of success in the two methodologies confirmed the advantage of GentleMACS in obtaining tumor cell lines $(p=0.015)$.

Table 1. Primary cultures from solid tumors disaggregated with cell scraper.

\begin{tabular}{cccc}
\hline Tumor type & Samples seeded & Tumor cell lines obtained & Success rate (\%) \\
\hline Kidney & 17 & 12 & 70.5 \\
Colon & 15 & 5 & 33.3 \\
Breast & 10 & 5 & 50 \\
Ovarian & 9 & 6 & 66.6 \\
Endometrial & 7 & 3 & 43 \\
Sarcoma & 6 & 4 & 66.6 \\
Melanoma & 6 & 2 & 33.3 \\
Gist $*$ & 4 & 2 & 50 \\
Mesothelioma & 1 & 1 & 100 \\
Neoplastic effusion & 5 & 5 & 100 \\
\hline
\end{tabular}

In the table are reported the success rate in expanding tumor cells starting from tumor samples after surgery. The rate of failure was $46 \%$. Failure was mainly due to bacterial or fungal contamination (31\%) of the original tumor sample or to inability of tumor cells to expand in vitro $(69 \%) ;{ }^{*}$ Gastrointestinal stromal tumor.

Figure 1. Growth potential of primary cell cultures from different solid tumors and neoplastic effusions (average of passages).

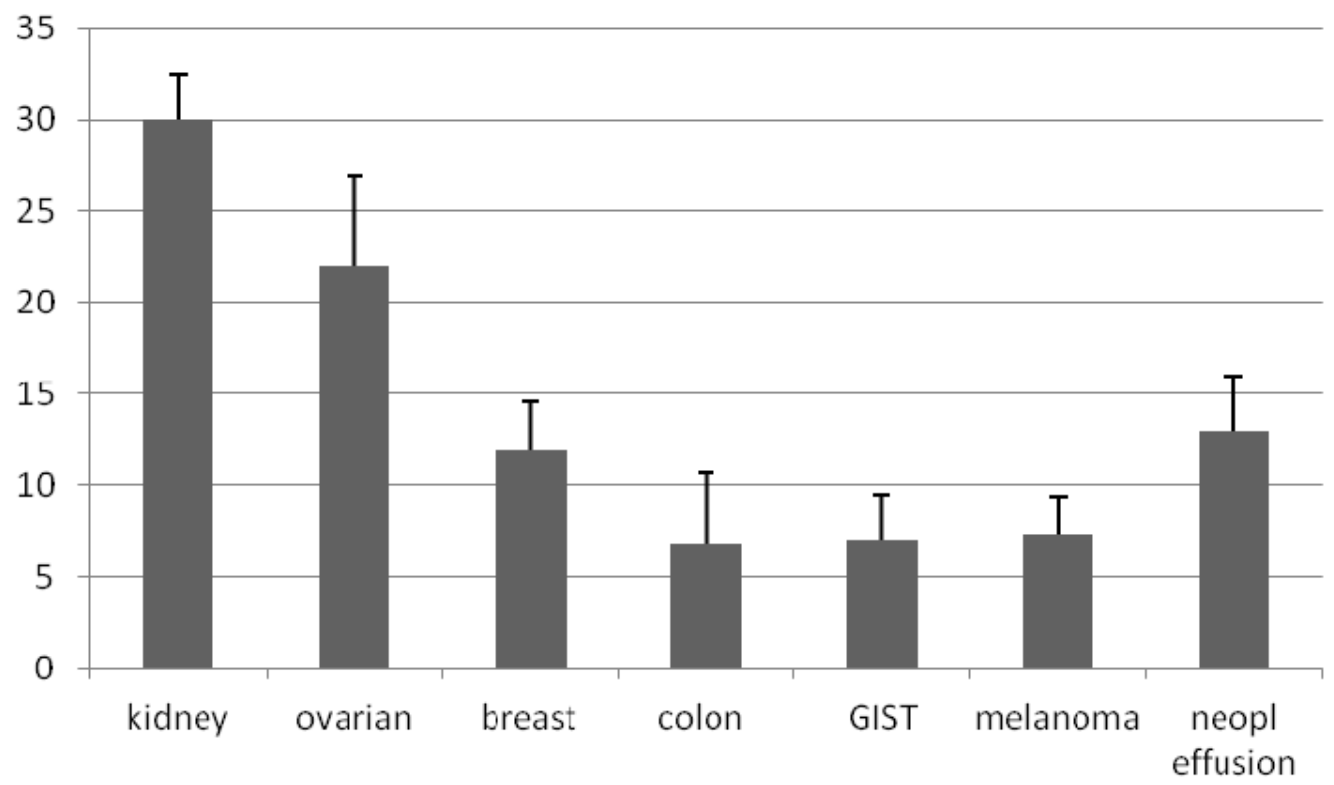

In the figure are reported the mean number of passages $+/-\mathrm{SD}$ of the first 40 tumor lines obtained with mechanical disaggregation following enzymatic treatment from different types of cancer. 
Table 2. Primary cultures from solid tumors disaggregated with GentleMacs.

\begin{tabular}{cccc}
\hline Tumor type & Samples seeded & Tumor cell lines obtained & Success rate (\%) \\
\hline Kidney & 6 & 4 & 66 \\
Colon & 25 & 20 & 80 \\
Ovarian & 3 & 2 & 66.6 \\
Sarcoma & 6 & 5 & 83.3 \\
\hline
\end{tabular}

40 tumor samples derived from primary or metastatic tumors were disaggregated with GentleMacs in a closed system and cultured under GMP conditions. In 31 out of 40 samples we were able to obtain tumor cell lines with pathologic characteristics in the majority of cells. The rate of failure was $22 \%$. Failure was mainly due to bacterial or fungal contamination $(55 \%)$ of the original tumor sample or to inability of tumor cells to expand in vitro $(44 \%)$.

\subsection{Ascite-Derived Ovarian Cancer Cells}

Ten ascitic fluid samples from different ovarian cancer patients were processed employing different techniques to determine the best approach for isolating and expanding ovarian cancer cells from ascites. When all cells present in the ascitic fluid were cultured, after a few passages, fibroblasts or mesothelial cells had a great growth advantage in comparison with tumor cells irrespective of the number of tumor cells present in the ascitic fluid. To overcome this problem, after determining the percent of CD326 cells present in the ascitic fluid, we performed positive selection with specific microbeads. Before separation, the mean number of CD326+ cells was $37 \%$ (range $1 \%-85 \%$ ). After enrichment, the mean positive cells increased to $80 \%$ (range $75 \%-95 \%$ ) (Table 3). Positive cells were immediately cryopreserved, if sufficient for establishing further experiments or alternatively used to establish primary tumor cell line cultures. We documented that the purification of CD326 cells allowed the establishment of cultures containing more than $90 \%$ tumor cells. Results obtained from flow cytometry analyses corresponded with the pathology results (data not shown).

Table 3. Primary ovarian tumor cells cultures established from ascitic fluid.

\begin{tabular}{|c|c|c|c|c|c|c|}
\hline \multirow{2}{*}{ Patients } & \multicolumn{2}{|c|}{ Before purification } & \multicolumn{2}{|c|}{ After purification } & \multirow{2}{*}{$\begin{array}{l}* \text { Tumor } \\
\text { cell lines }\end{array}$} & \multirow{2}{*}{$\begin{array}{l}\text { \ Tumor cells } \\
\text { cryopreserved }\end{array}$} \\
\hline & Cell count & \% CD326+ & Cell count & \% CD326+ & & \\
\hline AF001 & $30 \times 10^{6}$ & 3 & $5 \times 10^{4}$ & 70 & $\mathrm{ND}+$ & ND \\
\hline AF002 & $30 \times 10^{6}$ & 70 & $15 \times 10^{6}$ & 85 & ND & $15 \times 10^{6}$ \\
\hline AF003 & $30 \times 10^{6}$ & 82 & $20 \times 10^{6}$ & 90 & ND & $20 \times 10^{6}$ \\
\hline AF004 & $150 \times 10^{6}$ & 5 & $3 \times 10^{6}$ & 85 & ND & $3 \times 10^{6}$ \\
\hline AF005 & $3 \times 10^{6}$ & 2 & $1 \times 10^{4}$ & 80 & yes & $2 \times 10^{6}$ \\
\hline AF006 & $25 \times 10^{6}$ & 66 & $15 \times 10^{6}$ & 90 & ND & $15 \times 10^{6}$ \\
\hline AF007 & $82 \times 10^{6}$ & 4 & $2 \times 10^{6}$ & 88 & ND & $2 \times 10^{6}$ \\
\hline AF008 & $30 \times 10^{6}$ & 85 & $20 \times 10^{6}$ & 95 & ND & $20 \times 10^{6}$ \\
\hline AF009 & $13 \times 10^{6}$ & 4 & $30 \times 10^{4}$ & 75 & yes & $4 \times 10^{6}$ \\
\hline AF0010 & $10 \times 10^{6}$ & 50 & $12 \times 10^{6}$ & 95 & ND & $12 \times 10^{6}$ \\
\hline
\end{tabular}

* Tumor cell lines were established by culturing CD326-positive cells in complete medium as described in Material and Methods section; $\uparrow$ Tumor cells were freshly cryopreserved after CD326 purification, when the number of cells recovered was sufficient, or after five culture passages; $\$$ Not Done because the number of tumor cells obtained after disaggregation was sufficient to be cryopreserved for further experiments. 


\subsection{Characterization of Primary Cultures}

It was particularly important to determine whether the cells recovered were representative of tumor cells from the original tumor. Solid adenocarcinomas, like most of the tumors represented in this study, are epithelial in nature, and typically express cytokeratins in their cell cytoplasm. Moreover, monoclonal antibodies to cytokeratins stain the cytoplasm of epithelial but not fibroblast cells (the most important cell culture contaminant) [1]. Analysis of cultures revealed that in $>90 \%$ of samples, the prevalent cellular component of cultures, irrespective of disaggregation type employed, was of tumor origin. In five cultures derived from tumor samples disaggregated with a cell scraper, tumor cell enrichment using anti-fibroblast microbeads was required due to the presence of variable levels of fibroblast-like cells. In two selected cases (1 kidney and 1 ovarian), morphological and phenotypical examination of growing cultures at the 4th and 20th passage were compared, and revealed no differences between cultures (Figure 2).

Figure 2. Phenotypical analysis of two primary tumor cultures. Cytokeratin immunofluorescence analyses of primary cultures derived from ovarian tumor at 4th (A) and 40th (B) passage, and from a clear renal cell carcinoma at 4th (C) and 20th (D) passage (Magnification 200×).

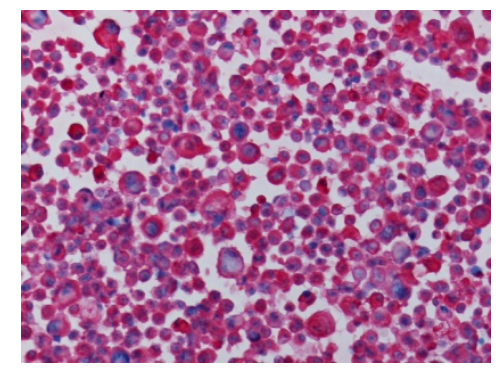

(A)

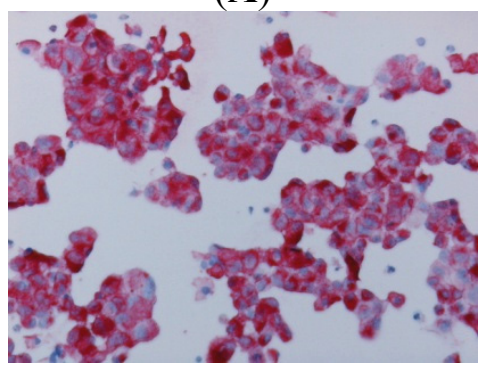

(C)

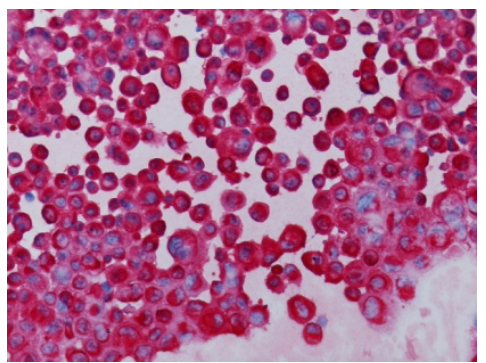

(B)

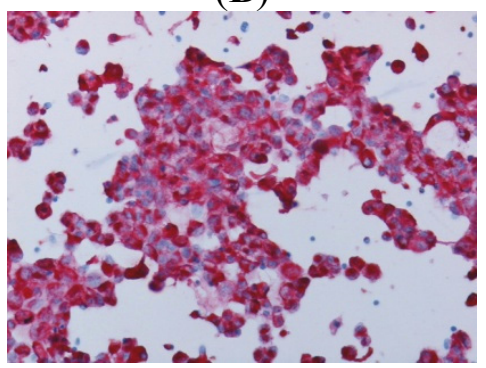

(D)

\section{Discussion}

Techniques to grow human cells and tissues on a plastic surface or in suspension have significantly contributed to the success of many biomedical investigations. Successful isolation and growth of tumor cells from freshly excised tumors, depends on several factors including receipt of tissue that contains a significant number of malignant cells for culture, the type of tumor and the in vitro manipulation, which may or may not result in a stable cell line. In general, the explant culture system is technically simpler, requiring almost no special experience or reagents, but fibroblasts will eventually overgrow tumor cells in conventional culture medium $[5,11]$. In addition, new growth in the culture is limited to 
cells that have migrated out from the initial tissue fragment. Recent data demonstrated that a large number of melanoma cell lines can be successfully obtained and characterized [12]. However, no data are available on the possibility of efficiently expanding tumor cells derived from different types of solid tumors. Here we describe a simple and rapid method for the establishment of primary cultures from solid tumors and neoplastic effusions. In the first group of experiments when tumor specimens were disaggregated with a cell scraper, in less than $50 \%$ of the clinical samples we were able to generate primary cultures that could be maintained for a significant number of passages in vitro. Even though the method we described is efficient in establishing primary culture from almost every type of solid tumor, the best results were obtained with kidney and ovarian tumors. Primary cultures were characterized by a number of evaluation criteria including morphological and immunological parameters to confirm that tumor-derived primary cultures retain the characteristics and behavior of malignant cells. These results support the concept that in short-term culture systems based on enzymatic-mechanical disaggregation of tumor specimens, it is possible to grow malignant cells from a wide variety of solid tumors and, in some cases, to obtain primary tumor cell lines.

In the early phase, cell cultures may contain a variable number of contaminating fibroblast-like cells; these usually disappear after a number of passages, but magnetic fibroblast depletion is required in a minority of cases. It is worth noting that cultures from almost all tumor types were initially adversely affected by the presence of trypsin in the enzymatic digestion mixture (data not shown), but this effect did not persist, even though the rate of growth was in general impaired.

Moreover, the efficiency in establishing and maintaining tumor cells in culture depends on the characteristics of the original sample, which in most cases could be rich in connective tissue. In addition with this methodology, in most cases small fragments were obtained and it was not possible to estimate how many cells were plated.

To overcome some of these problems, in a further set of experiments, we evaluated whether it was possible to obtain tumor cell lines using the GentleMACS instrument, which allows automated dissociation of tumor tissues into single-cell suspensions in a closed and sterile system. In preliminary experiments we demonstrated that in most cases tumor samples could be treated without previous digestion, while for samples requiring digestion a short time incubation before dissociation with collagenase was sufficient to obtain a suspension of cells. The use of enzymatic digestion could be a limitation, at least for some types of solid tumors, in that it has been described that culture of isolated cells from protease-digested solid tumors includes the risk of an overgrowth by fibroblasts or stromal cells [13]. In addition, according to the pathologist's indications we treated the portion of the tumor sample richest in tumor cells, resulting in highly efficiency obtainment of tumor cell lines without fibroblast contamination. Failure was mainly due to bacterial or fungal contamination of the original tumor sample or to an intrinsic inability of the tumor cells to expand in vitro. We are still working with surgeons and pathologists to reduce sample contamination. This methodology is very simple and reproducible, since after dissociation it is possible to filter the material and obtain a suspension of cells, cluster free, that can be counted and plated at an opportune concentration. For the clinical translational of this approach it is important to assess the molecular features of tumor cell lines. However, considering that several types of tumor were treated, and there was some difficulty in defining specific tumor-associated antigens, it was not possible to conduct a full molecular characterization of the tumor cell lines obtained in comparison with the primary tumor. So, in order to 
define the neoplastic origin of the expanding cells they were stained with haematoxylin-eosin to identify malignant cells on the basis of cytomorphology by pathologists.

Another matter of concern is the possibility of obtaining sizeable numbers of ovarian tumor cells from ascitic fluid even when they are initially present at a low percentage. For this reason, an efficient method for enriching and purifying ovarian tumor cells would facilitate tumor cell line cultures. It has been demonstrated that EpCAM represents an optimal antigenic target for separation of malignant cells of epithelial origin, as it is expressed on the great majority of epithelial tumor cells and does not react with fibroblasts, mesothelial cells or other cells types that are usually present in ascitic fluid of ovarian cancer patients [14]. Our results clearly demonstrate that the growth of contaminating cells present in ascitic fluid can overgrow malignant cells present in the specimens, even if initially present at high percentages. By using specific microbeads directed against CD326 (EpCam) we were able to positively select malignant cells and to clear the majority of contaminants. The cells recovered were highly enriched CD326 cells, even when they were present in a very low percentage in the original sample. Of note in most cases positive cells recovered after enrichment were sufficient for cryopreservation for later use, otherwise, when the collected amount was low, cell culture allowed obtainment of ovarian tumor cell lines that maintained the characteristics of the original tumor and reached a consistent number of malignant cells.

\section{Conclusions}

Overall the methodology described here presents several advantages including the simplicity of culture maintenance, purity of the cell populations, possibility to obtain sufficient number of tumor cells after only a few culture passages, as well as preservation of the morphological and phenotypical characteristics of the original tumor.

Cell lines generated by this method may prove useful, as in vitro models for anticancer drug testing/development, and for facilitating the clinical development of CTL-based immunotherapeutic strategies based on the use of vital tumor cells as source of tumor antigens [8,14-17]. As previously demonstrated by our group, treatment with specific autologous antitumor CTLs in vitro generated induced immunological responses and long-lasting clinical benefit in a patient with RCC [18]. Using, as stimulator cells, tumor cells obtained with the approach here described, is safe and in an ongoing study at our institution we will define, in a larger series of patients, whether this form of immunotherapy has a role in advanced pre-treated cancer patients failing conventional therapies.

\section{Acknowledgements}

This work was partially supported by grants from the Ministero della Salute (to DM) and by the Fondazione IRCCS Policlinico San Matteo Pavia Ricerca Corrente to (DM and PP).

\section{Author Contributions}

Study design: DM, RS, and PP. Manuscript writing and data analysis: DM and RS. Provision of tumor samples: MM, PD, MR and AS. Expansion and characterization of tumor cell lines:IT, FF and MT. Isto-pathological analysis: OL, BDB and MP. Manuscript critical reading: RM and PP. 


\section{Conflicts of Interest}

The authors declare no conflict of interest.

\section{References}

1. Ochs, R.L.; Fensterer, J.; Ohori, N.P.; Wells, A.; Gabrin, M.; George, L.D.; Kornblith, P. Evidence for the isolation, growth and characterization of malignant cells in primary cultures of human tumors. In Vitro Cell. Dev. Biol. Anim. 2003, 39, 63-79.

2. Bonner-Weir, S.; Taneja, M.; Weir, G.C.; Tatarkiewicz, K.; Song, K.H.; Sharma, A.; O’Neil J.J. In vitro cultivation of human islets from expanded ductal tissue. Proc. Natl. Acad. Sci. USA 2000, 97, 7999-8004.

3. Dairkee, S.H.; Paulo, E.C.; Traquina, P.; Moore, D.H.; Ljung, B.M.; Smith, H.S. Partial enzymatic degradation of stroma allows enrichment and expansion of primary breast tumor cells. Cancer Res. 1997, 57, 1590-1596.

4. Croce, M.V.; Colussi, A.G.; Segal-Eiras, A. Assessment of methods for primary culture of human breast epithelia. J. Exp. Clin. Cancer Res. 1998, 17, 19-26.

5. Chen, L.L.; Mann, E.; Greenberg, B. Removal of fibroblast from primary cultures of squamous cell carcinoma of the head and neck. J. Tissue Cult. Methods 1993, 15, 1-10.

6. Tveit, K.M.; Pihl, A. Do cell lines in vitro reflect the properties of the tumours of origin? A study of lines derived from human melanoma xenografts. Br. J. Cancer 1981, 44, 775-786.

7. Schiavo, R.; Tullio, C.; la Grotteria, M.; Andreotti, I.C.; Scarpati, B.; Romiti, L.; Bozzi, F.; Pedrazzoli, P.; Siena, S. Establishment and characterization of a new Ewing's sarcoma cell line from a malignant pleural effusion. Anticancer Res. 2007, 27, 3273-3278.

8. Montagna, D.; Schiavo, R.; Gibelli, N.; Pedrazzoli, P.; Tonelli, R.; Pagani, S.; Assirelli, E.; Locatelli, F.; Pession, A.; Fregoni, V.; et al. Ex vivo generation and expansion of anti-tumor cytotoxic T-cell lines derived from patients or their HLA-identical sibling. Int. J. Cancer 2004, 110, 76-86.

9. Parmiani, G.; de Filippo, A.; Novellino, L.; Castelli, C. Unique human tumor antigens: Immunobiology and use in clinical trials. J. Immunol. 2007, 178, 1975-1979.

10. Hayashi, K.; Yonamine, K.; Masuko-Hongo, K.; Iida, T.; Yamamoto, K.; Nishioka, K. Clonal expansion of $\mathrm{T}$ cells that are specific for autologous ovarian tumor among tumor-infiltrating $\mathrm{T}$ cells in humans. Gynecol. Oncol. 1999, 74, 86-92.

11. Moritake, H.; Sugimoto, T.; Kuroda, H.; Hidaka, F.; Takahashi, Y.; Tsuneyoshi, M.; Yoshida, M.A.; Cui, Q.; Akiyoshi, K.; Izumi, T.; et al. Newly established Askin tumor cell line and over expression of focal adhesion kinase in Ewing sarcoma family of tumors cell lines. Cancer Genet. Cytogenet. 2003, 146, 102-109.

12. Behren, A.; Anaka, M.; Lo, P.H.; Vella, L.J.; Davis, I.D.; Catimel, J.; Cardwell, T.; Gedye, C.; Hudson, C.; Stan, R.; et al. The Ludwig Institute for Cancer research Melbourne Cell line panel. Pigment Cell Melanoma Res. 2013, 26, 597-600.

13. Hass, R.; Bertram, C. Characterization of human breast cancer epithelial cells (HBCEC) derived from long term cultured biopsies. J. Exp. Clin. Cancer Res. 2009, 28, 127. 
14. Went, P.T.; Lugli, A.; Meier, S.; Bundi, M.; Mirlacher, M.; Sauter, G.; Dirnhofer, S. Frequent EpCam protein expression in human carcinomas. Hum. Pathol. 2004, 35, 122-128.

15. Turin, I.; Pedrazzoli, P.; Tullio, C.; Montini, E.; la Grotteria, M.C.; Schiavo, R.; Perotti, C.; Locatelli, F.; Carretto, E.; Maccario, R.; et al. GMP production of anti-tumor cytotoxic T-cell lines for adoptive T-cell therapy in patients with solid neoplasia. Cytotherapy 2007, 9, 499-507.

16. Dudley, M.E.; Yang, J.C.; Sherry, R.; Hughes, M.S.; Royal, R.; Kammula, U.; Robbins, P.F.; Huang, J.; Citrin, D.E.; Leitman, S.F.; et al. Adoptive cell therapy for patients with metastatic melanoma: Evaluation of intensive myeloablative chemoradiation preparative regimens. J. Clin. Oncol. 2008, 26, 5233-5239.

17. Montagna, D.; Turin, I.; Schiavo, R.; Montini, E.; Zaffaroni, N.; Villa, R.; Secondino, S.; Schiavetto, I.; Caliogna, L.; Locatelli, F.; et al. Feasibility and safety of adoptive immunotherapy with ex-vivo generated autologous, cytotoxic $\mathrm{T}$ lymphocytes, in patients with solid tumors. Cytotherapy 2011, 14, 80-90.

18. Secondino, S.; Turin, I.; Montini, E.; Porta, C.; Maccario, R.; Pedrazzoli, P.; Montagna, D. Case Report: Long lasting response in a patient with metastatic renal cell cancer receiving anti tumor cytotoxic T lymphocytes. Tumori 2013, 99, 282e-284e.

(C) 2014 by the authors; licensee MDPI, Basel, Switzerland. This article is an open access article distributed under the terms and conditions of the Creative Commons Attribution license (http://creativecommons.org/licenses/by/3.0/). 